Article

\title{
The Impact of Routine Skin Care on the Quality of Life
}

\author{
Lixia Zhang ${ }^{1}$, Aldhel Adique ${ }^{1}$, Pradipta Sarkar ${ }^{1}$, Vinay Shenai ${ }^{1}$, Murali Sampath ${ }^{2}$, Ricky Lai ${ }^{1}$, \\ Joanna $\mathrm{Qi}^{3}$, Macia Wang ${ }^{3}$ and Miranda A. Farage ${ }^{2, *(1)}$ \\ 1 The Procter and Gamble Company, International Operations SA Singapore Branch, Singapore 138547, \\ Singapore; zhang.1.27@pg.com (L.Z.); adique.ml@pg.com (A.A.); sarkar.p@pg.com (P.S.); \\ shenai.v@pg.com (V.S.); lai.rp@pg.com (R.L.) \\ 2 The Procter and Gamble Company, Mason, OH 45040, USA; sampath.m@pg.com \\ 3 The Procter and Gamble Company, Beijing 101312, China; qi.jo@pg.com (J.Q.); wang.zy.2@pg.com (M.W.) \\ * Correspondence: farage.m@pg.com
}

Received: 12 June 2020; Accepted: 21 July 2020; Published: 24 July 2020

check for updates

\begin{abstract}
Importance: Consumers purchase a wide variety of consumer products and come into contact with these products on a daily basis. Manufacturers invest deeply in developing new products or improving existing products, in order to produce a positive impact on the lives of consumers. Objective: The goal of this study was to determine the impact of over-the-counter skin care products on the quality of life (QoL) of female consumers. Design and Measures: A QoL instrument developed for consumer products (the Farage QoL with an added Skin Care Module) was used to assess the impact of a 28-day facial skin care regimen using commercially available products formulated to improve elasticity, firmness and hydration, and to correct age- and sun-related skin color. Responses were collected prior to study commencement, at completion of the product usage stage, and after a period of withdrawal of the product with reversion to a basic skin care regimen. Participants: Two main study groups from Australia included 89 new mothers, i.e., women with children 2 years and under (mean age \pm SD was $34 \pm 4.8$ ), and a national representative sample of 91 women $(45 \pm 12)$. An additional test group from China consisted of 40 younger cosmetic users (25 \pm 4.3$)$. The Skin Care Module was not included in the instrument for the third group. Results: After 28-days of usage, both test groups in the main study showed significant improvement in three of five items in the Skin Care Module (improved feelings of empowerment, happiness and self-esteem). Improvements persisted after 2 weeks of product withdrawal. In the main QoL instrument, the New Mothers group showed significant improvement in the Well-Being domain, driven by improvements in the Self-Image and Self Competence subdomains. The National Representative group showed improvements in the Energy and Vitality domain, driven by improvements in the Personal Pleasure, Physical State and Routine Activity subdomains. The additional group in the China study showed results similar to the New Mothers group. Conclusions and Relevance: A quality and efficacious skin care regimen can have a positive impact on the QoL of consumers. Differences in responses of the test groups were likely related to differences in the mean age and differences in time available to look after themselves.
\end{abstract}

Keywords: quality of life; farage quality of life; skin care; cosmetics

\section{Introduction}

Health related quality of life $(\mathrm{HRQOL})$ instruments have become an important part of evaluating interventions and treatment options in individuals suffering from a wide variety of medical conditions [1]. More recently, this approach has been expanded to evaluate the state of well-being of caregivers of individuals with serious medical or mental challenges. Examples are caregivers 
for individuals suffering from Parkinson's disease, patients with cancer, or individuals with schizophrenia [2-4]. Quality of life assessments are becoming widely used in dermatological practice [5], with a wide array of instruments developed for various disease states including acne, rosacea, atopic dermatitis, and vitiligo. Some investigators have used quality of life (QoL) measures to evaluate the patients' "willingness to pay", i.e., the perceived cost/benefit of treatment to the patient [6-8].

Despite the broad use of health related QoL, very few instruments have been developed to evaluate the impact of consumer products on quality of life. Consumers purchase a wide variety of products and come into contact with these products on a daily basis. Manufacturers invest deeply in developing new products or improving existing products in order to produce a positive impact on the lives of consumers. Developers of consumer products can benefit greatly from QoL instruments to define product attributes that would be valued by the consumer. However, evaluating the impact of consumer products on QoL presents a unique challenge. With health related QoL, the subjects in question are patients whose quality of life is likely lower than average as a result of a particular health challenge. The goal is to restore these individuals to a normal QoL that is comparable to the general population, e.g., to move the QoL from "bad to normal". With consumer products the individuals in question are already at the QoL of the general population, and the goal is to improve the QoL to a place above average, e.g., to move the QoL from "normal to better".

There are a few examples of instruments designed for evaluating the impact on QoL of specific consumer products. Beresniak and colleagues developed a QoL instrument to evaluate cosmetic products and physical appearance [9]. The final instrument consisted of 43 -items in five dimensions, and was validated in 13 countries among 3231 subjects. Chang and colleagues evaluated the performance of an over-the-counter skincare regimen in treating chronic xerosis and its impact on the subjects' quality of life using an 11-item instrument [10]. Clinically, subjects exhibited a demonstrable improvement in corneometry and D-Squame tape assessments, while perceiving a significant reduction in itchiness, other xerosis associated symptoms, and emotional impact. The impact on quality of life among women with hair loss was evaluated by Zhuang and colleagues [11]. At the first visit, subjects' quality of life was inversely related to their perception of the severity of hair loss via visual assessment scales. Subjects were re-tested after 12 months of treatment with twice daily applications of $2 \%$ minoxidil average scores for the group improved for both indices.

In another example of application of a QoL instrument developed for consumer products, Getliffe and colleagues developed QoL measures for absorbent products for incontinence [12]. These investigators proposed that results on the relative importance of product characteristics can be used to provide guidance toward developing products that improve comfort and odor containment, and better meet the needs of the consumer with regard to discretion and fit.

The Farage Quality of Life (FQoL $\left.{ }^{\mathrm{TM}}\right)$ instrument was developed to assess the potential impact of a broader range of consumer products on the quality of life [13]. The 27-item self-administered questionnaire covers a variety of elements pertaining to overall well-being, energy and vitality. In addition, modules can be added pertinent to specific categories of consumer products. Previously we published results of this QoL instrument with an added Menstrual Module, to evaluate the impact of menstrual control products on consumers' quality of life [13,14]. Results of a study conducted using this instrument determined that scores on items relating to well-being, emotional and physical state were lower during menstruation vs. before menstruation. However, if subjects were given a new, better performing menstrual pad scores did not worsen during menstruation.

Skin care products represent a large category of consumer products. In 2018, sales of skincare products in the US grew by $13 \%$ to an estimated USD 5.6 billion [15]. Recent decades have seen the emergence of cosmeceuticals, or products that have therapeutic effects capable of affecting the condition of the skin beyond the duration of application [16]. The effectiveness of such skin care products can be evaluated using a number of measures. Instruments are readily available to measure improvements in skin elasticity and firmness, increases in hydration, improvements in barrier function and hyperpigmentation. However, an additional important measure to determine the overall 
effectiveness of skin care products is the effect(s) of such products on the self-esteem and quality of life of the consumer.

The goal of this study was to determine the potential impact of over-the-counter skin care products on the quality of life of female consumers by evaluating product benefits in addition to technical benefits. Quality consumer products can contribute to overall well-being, and an increase in various aspects of QoL during use providing holistic product benefits to meet the overall needs of our consumers. Participants were asked to use specific skin care products for 28 -days. The key active in the skin care products is niacinamide. This material is known to be effective in improving skin texture, elasticity, fine lines and wrinkles, red blotchiness, hyperpigmentation, sun damage and sallowness seen with ageing $[17,18]$. Although objective data are not presented in this manuscript, our own data in the study conducted in China and described below showed significant improvement in skin hydration, skin barrier function, and skin elasticity after 8 days of product use. Objective measured conducted 5 days after product was withdrawn, demonstrated continued improvement over pre-use levels (unpublished data).

Using the FQoL ${ }^{\mathrm{TM}}$ instrument, the impact of the skin care regimen on quality of life was evaluated before and after completion of the product use, and several days after cessation of product use.

\section{Methods}

In the main study conducted in Australia, participants were asked to use a basic skin care regimen for 3 days consisting of a placebo ( $3 \%$ glycerin cream with no actives) as their only skin care product (washout period). This was followed by a 28-day test period, where participants were asked to use a skin care regimen twice a day. During this product use phase test products were identified only as "Facial essence", "Facial cream", and "Eye cream". Brand names were not disclosed, and neither the testing facility nor the participants were aware of the product identity. After the use phase subjects returned to the basic skin care regimen using the placebo (product deprivation period). The skin care regimen in the product use phase consisted of three commercially available products (Olay ${ }^{\circledR}$ products, Procter \& Gamble, Australia) including a tone-correcting serum (Luminous Tone Perfecting Treatment), a wrinkle-smoothing cream (Regenerist Micro-Sculpting Cream) and a color-correcting eye cream (Ultimate eye cream). Participants were asked to complete the FQoL ${ }^{\text {TM }}$ instrument after the washout period (i.e., baseline), after using the test products for 28 days, and after the 14-day product deprivation period. Study participants were recruited through a local, independent testing agency. The study groups consisted of mothers with children 2 years old and under (i.e., New Mothers), and a National Representative sample (Table 1). Among the New Mothers group, 89 subjects completed the 28-day skin care regimen phase and 83 subjects went on to 42 days to complete the deprivation phase. Among the National Representative group, 91 subjects completed the 28-day skin care regimen phase and 90 subjects completed the deprivation phase.

Table 1. Demographics of Study Groups.

\begin{tabular}{|c|c|c|c|}
\hline & \multicolumn{2}{|c|}{ Australia Study } & China Study \\
\hline & New Mothers ${ }^{a}$ & National Rep ${ }^{b}$ & Younger Cosmetic Users \\
\hline Mean Age $( \pm \mathrm{SD})$ & $34( \pm 4.8)$ & $45( \pm 12.0)$ & $25( \pm 4.3)$ \\
\hline Age Range & $22-50$ & $22-65$ & $20-33$ \\
\hline \# completing use phase & 89 & 91 & 40 \\
\hline \# completing deprivation phase & 83 & 90 & 40 \\
\hline
\end{tabular}

The FQoL ${ }^{\mathrm{TM}}$ was modeled initially after the World Health Organization general quality of life instruments [19], using a categorical scale of 1-5. The validated FQoL ${ }^{\mathrm{TM}}$ instrument used in this study consisted of 27 items, covering overall quality of life (1 item), and two other domains: Well-Being 
(12 items) and Energy and Vitality (14 items) [13]. The two domains are subdivided into a total of six subdomains, as shown in Table 2. All items were scored on a 5-point Likert scale, asking responders their level of agreement with each statement (strongly agree, agree, neither agree nor disagree, disagree, strongly disagree). Responses were converted into numerical values $1-5$, such that the most positive responses were assigned the higher numbers. In addition to the standard FQoL ${ }^{\mathrm{TM}}$ instrument a Skin Care Module was included, consisting of 5 additional items specifically targeted toward the participants' feelings toward their skin condition (Table 2).

Table 2. Structure of the Farage Quality of Life (FQoL $\left.{ }^{\mathrm{TM}}\right)$ Instrument.

\begin{tabular}{|c|c|c|c|}
\hline Domains & Sub-Domains & Number of Items & Definition \\
\hline \multicolumn{2}{|c|}{ Quality of Life } & 1 & Overall quality of Life \\
\hline \multirow{3}{*}{ Well-Being } & Emotion & 5 & Reflects a positive outlook on life \\
\hline & Self-Image & 5 & $\begin{array}{c}\text { Reflects self-acceptance, self-confidence and physical } \\
\text { appearance }\end{array}$ \\
\hline & Self-Competence & 2 & Reflects self-competence \\
\hline \multirow[b]{2}{*}{ Energy and Vitality } & Personal Pleasure & 7 & Includes the things consumers do for leisure and pleasure. \\
\hline & Physical State & 4 & $\begin{array}{c}\text { Involves physical activities, energy, household activities } \\
\text { consumers do. }\end{array}$ \\
\hline \multicolumn{2}{|c|}{ Skin Care Module } & 5 & $\begin{array}{l}\text { Questions on empowerment, self-esteem, confidence, } \\
\text { attractiveness, and happiness with facial skin conditions. }\end{array}$ \\
\hline
\end{tabular}

For each test group, the responses at baseline were compared to responses at 28-days after completion of the product use phase, and at 42-days after 14 days of product deprivation. The change in the response for each individual item was evaluated for statistical significance using a non-parametric Wilcoxon signed rank test. The Pratt method was used to address zero values [20]. The subdomains and domains were averages of responses to individual items within that grouping, and means were evaluated using a $t$-test. The 2 -sided $p$ values are reported. All analyses were done using JMP Pro version 14.2.0-Statistical Discovery Software from SAS.

A similar study was conducted in China using a group of young women recruited through a local, independent testing agency. (See Table 1 for demographic information.) The study was designed to evaluate a similar unidentified skin care regimen consisting of a cleanser, a clear lotion, an essence serum, and a moisturizer (Olay ${ }^{\circledR}$ Golden Aura products, Procter \& Gamble, China). After a 3-day wash-out period was completed, the products were used under a clinical setting for 28-days. This was followed by a 5-day product deprivation phase. Previously, a Chinese language version of the FQoL ${ }^{\mathrm{TM}}$ was translated and validated [21]. This instrument was administered at baseline, after completion of the use phase at 28-days, and after the 5-day deprivation phase at 33-days. The Skin Care Module was not included in the study conducted in China. No correction was made for multiple comparisons, as, typically, quality of life studies are not confirmatory studies focusing on a small number of end points. However, we obtained more than statistically significant results in 39 out of a total of 128 statistical tests (32 questions $\times 2$ segments $\times 2$ time points), which is much higher than $5 \%$, as expected by random chance.

\section{Results}

In the study conducted in Australia, an additional Skin Care Module was included as a part of the FQoL ${ }^{\mathrm{TM}}$ instrument (questions shown in Table 2). Figure 1 presents results for the two cohorts: New Mothers (a), and National Representative (b). After the 28-day product usage period both test groups showed significant improvement over baseline when asked if they felt empowered and happy with the condition of their skin. In addition, both groups responded more favorably when asked if the condition of their skin improved their self-esteem. For all three of these questions the improvement persisted 2 weeks after product usage ceased (day 42). 
The New Mothers group also showed significant improvement at the completion of the 28-day usage period and the follow up 14 days of deprivation period (day 42) in the level of confidence without make-up ( $p$-values $=0.002$ and 0.025 , respectively) (Figure $1 \mathrm{a}$ ). For this question, the National Representative group (Figure 1b) did not show significant improvement at the either time point. However, this group showed improvement when asked if the condition of their skin made them feel more attractive at the 28- and 42-day time point ( $p=0.003$ and 0.001 , respectively) (Figure $1 \mathrm{~b}$ ).

Figure 2 presents results of the FQoL ${ }^{\mathrm{TM}}$ among the new mothers with children 2 years or younger. The mean score for the Well-Being domain increased significantly at day 28, after one month of use of the three-part skin care regimen $(p=0.013)$, and continued to improve after the 2-week product deprivation period $(p=0.002)$ (Figure $2 \mathrm{a}$ ). The means for the Overall QoL and for the Energy and Vitality domain did not improve significantly at either time point. Figure $2 b$ demonstrates that improvement in the Well-Being domain scores was driven by significant increases in scores for the Self-Image and Self Competence subdomains. In both subdomains improvement continued through the product deprivation period (i.e., at the 42-day time point). Within the Energy and Vitality domain responses in Personal Pleasure improved significantly after the 28 days of product use $(p=0.025)$. Improvement in Physical State was significant at day 42 after the 2 weeks deprivation period $(p=0.051)$.

In the National Representative cohort, the mean score for Overall QoL or the Well-Being domain did not increase significantly (Figure 3a). However, responses in the Energy and Vitality domain showed significant improvement after 4 weeks of the skin care regimen (Day 28, $p=0.001$ ) (Figure 3a). The increase persisted after the 2-week product deprivation period (Day 42, $p=0.028$ ). The increase in the Energy and Vitality domain at 28 days was driven by significant increases in mean scores for all three subdomains, i.e., Personal Pleasure, Physical State, and Routine Activity (Figure 3b). After the 2-week deprivation period (Day 42) the Physical State mean score was still significantly elevated over the baseline $(p=0.011)$.

Individual items in the FQoL ${ }^{\mathrm{TM}}$ instrument were also evaluated for significant differences. The mean changes from baseline for each group at both 28- and 42-days were analyzed (Table 3). In the New Mothers group, most of the significant improvements were in the Well-Being domain. In the Self-Image subdomain, feeling good about oneself improved at day $42(p=0.004)$, self-confidence shows significant improvements at both 28-day and 42-day time point ( $p=0.034$ and 0.037 , respectively); and feelings about physical appearance show significant improvements at both 28-day and 42-day time point ( $p=0.001$ and 0.002 , respectively). In the Self-Competence subdomain significant improvement was noticed at day 42 with feelings about the participants' ability to take care of themselves showing improvement at day $42(p<0.001)$. The item about feeling confident showed improvement at both 28 and 42-days ( $p=0.009$ and 0.003 , respectively).

In specific items in the FQoL ${ }^{\mathrm{TM}}$ the National Representative group showed significant improvements in self-confidence within the Self-Image subdomain at both day 28 and day $42(p=0.034$ and 0.043 , respectively) and physical appearance at day $42(p=0.001)$ (Table 3). However, most of the improvements in means for individual items of this group were in the Energy and Vitality domain. Responses indicated improvement in the ability to attend public functions ( $p=0.033$ at day 42 ), engage in enjoyable activities ( $p=0.034$ at day 28), and enjoy being with friends and family $(p=0.008$ and 0.006 at days 28 and 42, respectively). Among the four items in the Physical State subdomain having more energy showed statistically significant improvement at day $28(p=0.01)$ and both feeling less tired and ability of complete household chores showed statistically significant improvement at day 42 ( $p=0.018$ and 0.022 , respectively). 


\section{a. New Mothers}

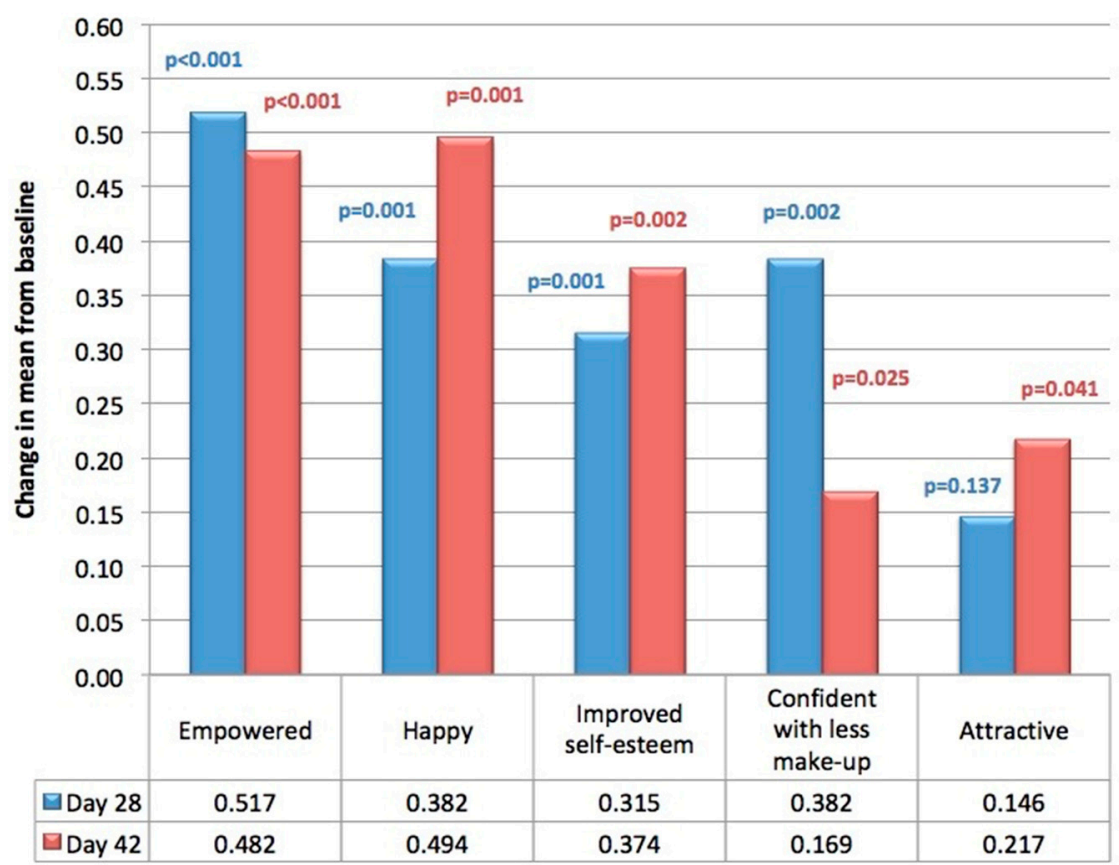

b. National Representative

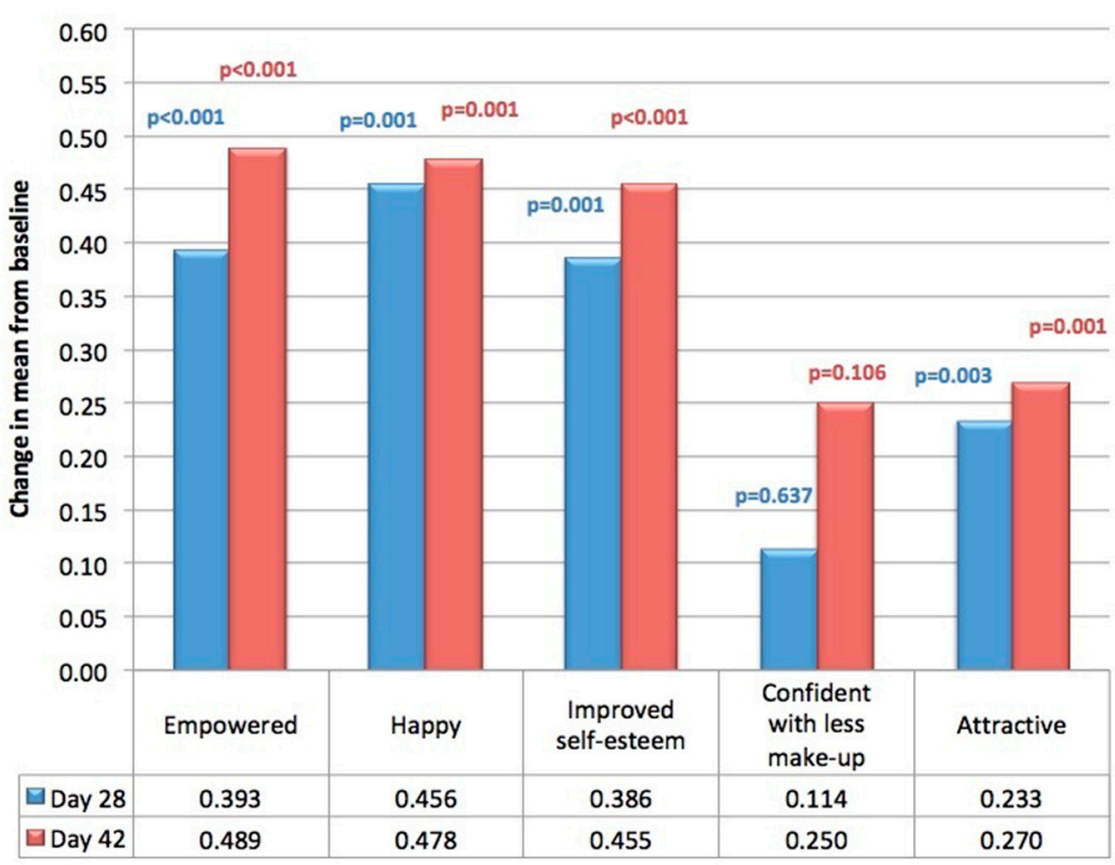

Figure 1. In a study of a three-part skin care regimen, participants were asked to fill out a Skin Care Module consisting of five questions that was included as a part of the FQoL ${ }^{\mathrm{TM}}$ instrument. Mean scores for each item were determined at three time points: before study commencement (i.e., baseline), after a 4-week usage period (day 28), and after 2-weeks of product deprivation (day 42). The 28- and 42-day mean changes from baseline are shown. The change in the response for each individual item was evaluated for statistical significance using a non-parametric Wilcoxon signed rank test. The 2-tailed $p$ values are reported. (a) New Mothers group, and (b) National Representative group. 


\section{a. New Mothers - Domains}

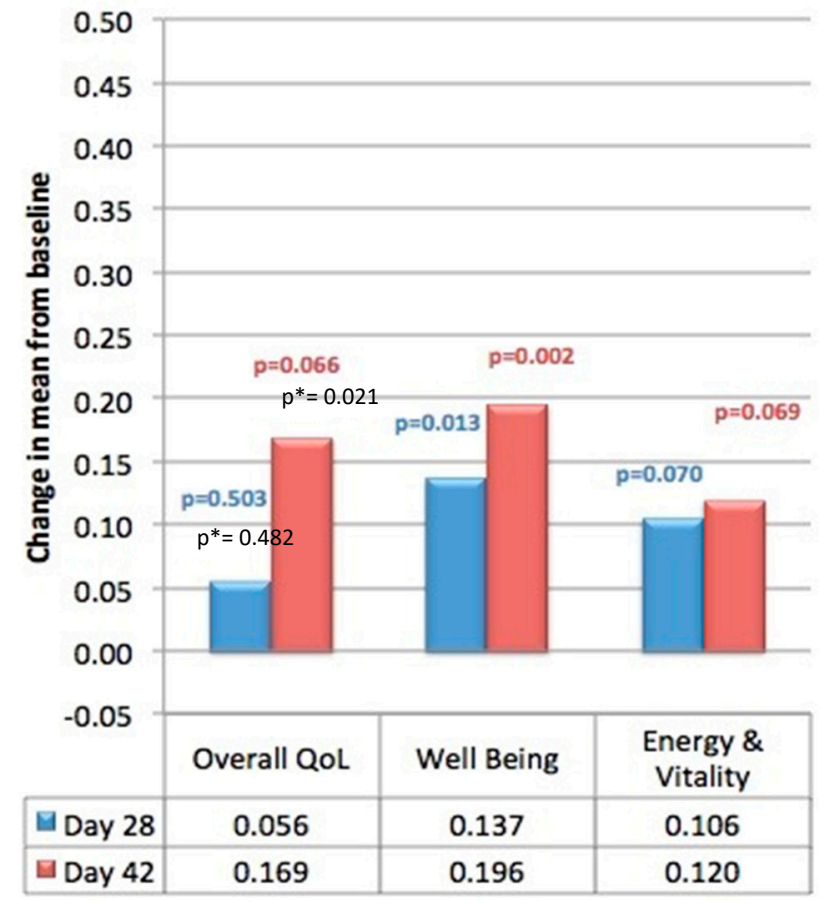

\section{b. New Mothers - Subdomains}

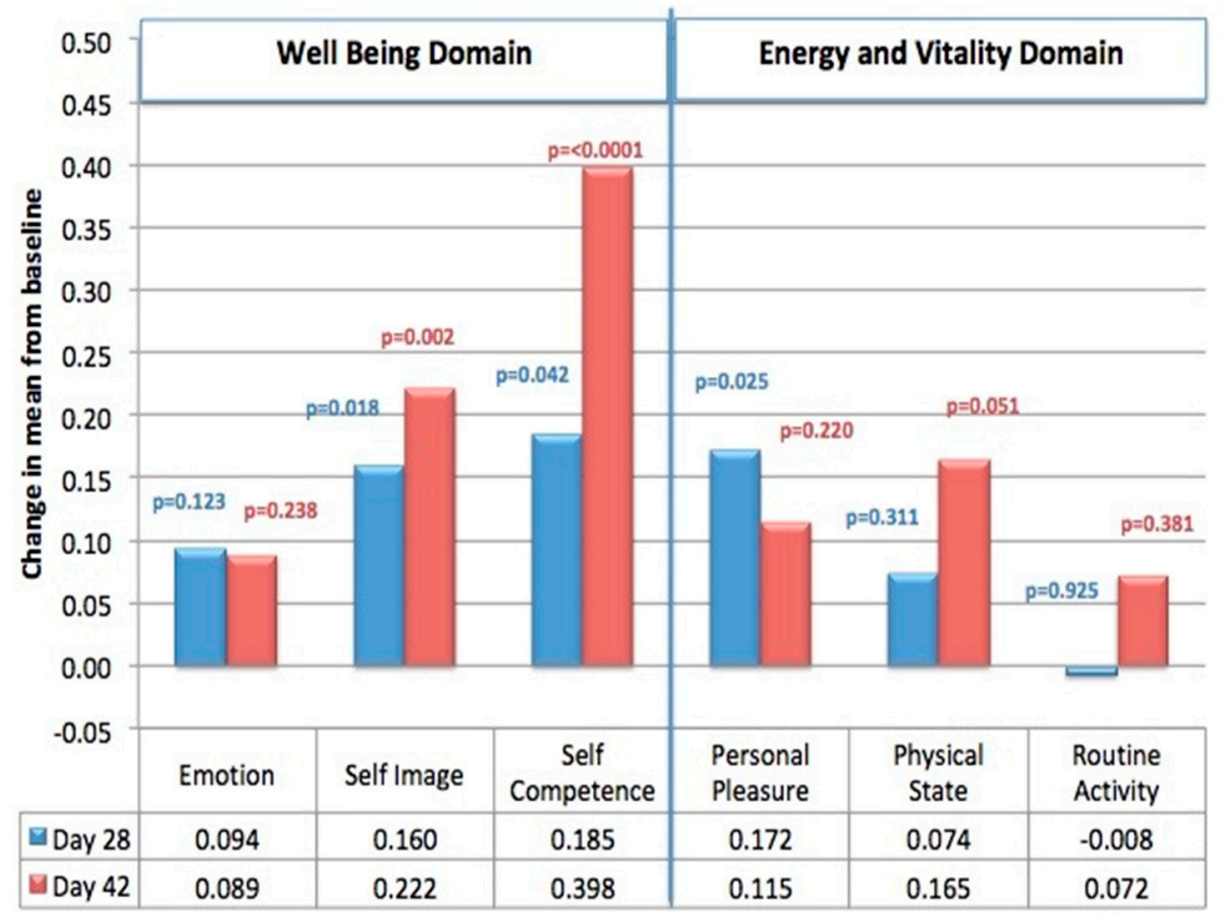

Figure 2. The FQoL ${ }^{\mathrm{TM}}$ instrument was administered at three total time points. Mean scores for domains (a) and subdomains (b) were determined at 28- and 42-days and compared to the baseline means for significant changes using a $t$ test. The 2-tailed $p$ values are reported. In this group, 89 participants completed day 28 , and 83 completed day 42. For Overall QoL, the $p$-values from non-parametric tests are also reported and denoted by $p^{*}$. 
a. National Representative - Domains

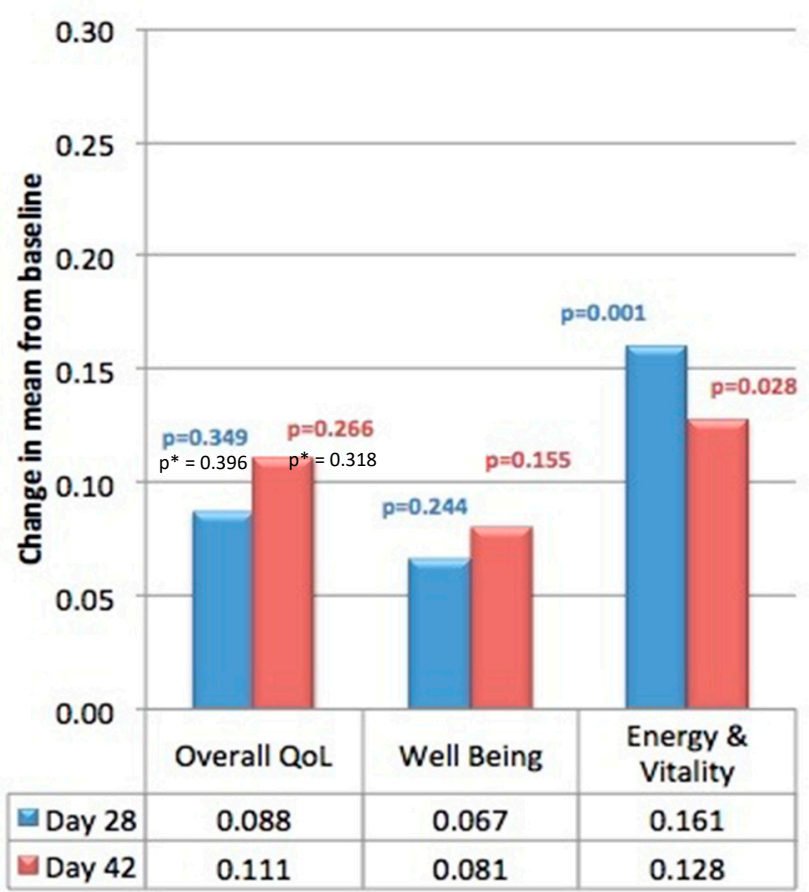

\section{b. National Representative - Subdomains}

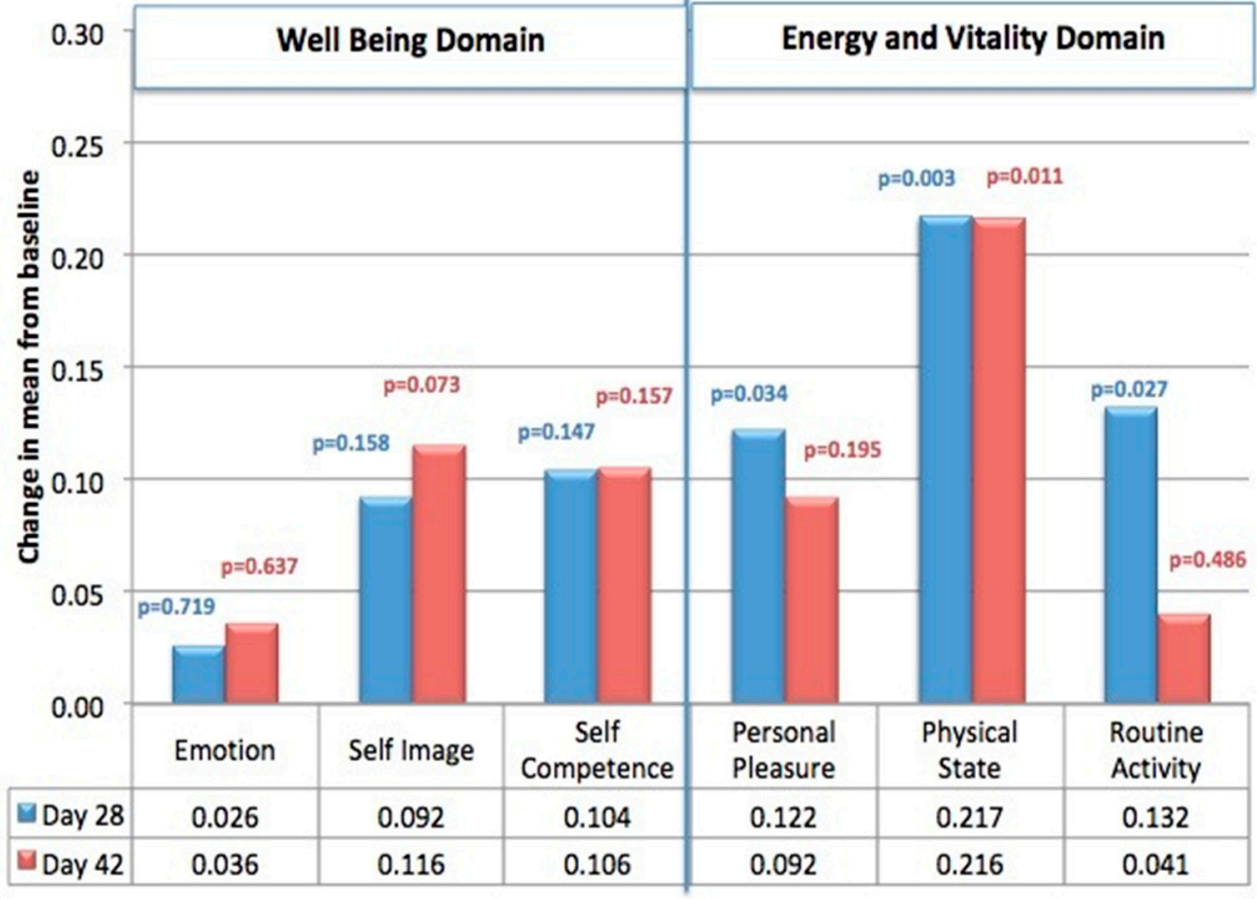

Figure 3. Mean changes in scores for the domains (a) and subdomains (b) are shown. In this group, 91 participants completed day 28 , and 90 completed day 42. The 2-tailed $p$-values from $t$-test are reported. For overall quality of life $(\mathrm{QoL})$, the $p$-values from non-parametric tests are also reported and denoted by $p^{*}$. 
Table 3. Mean change in responses to individual questions in Australia study.

\begin{tabular}{|c|c|c|c|c|c|c|c|c|}
\hline & \multicolumn{4}{|c|}{ New Mothers $(\mathrm{N}=89)$} & \multicolumn{4}{|c|}{ National $\operatorname{Rep}(\mathrm{N}=91)$} \\
\hline & \multicolumn{2}{|c|}{28 Days } & \multicolumn{2}{|c|}{42 Days } & \multicolumn{2}{|c|}{28 Days } & \multicolumn{2}{|c|}{42 Days } \\
\hline & $\begin{array}{c}\text { Mean } \\
\text { Change }\end{array}$ & $p$ value & $\begin{array}{c}\text { Mean } \\
\text { Change }\end{array}$ & $p$ value & $\begin{array}{c}\text { Mean } \\
\text { Change }\end{array}$ & $p$ value & $\begin{array}{l}\text { Mean } \\
\text { Change }\end{array}$ & $p$ value \\
\hline Overall Quality of Life & 0.056 & 0.482 & 0.169 & 0.021 & 0.088 & 0.396 & 0.111 & 0.318 \\
\hline \multicolumn{9}{|l|}{ Well-being Domain } \\
\hline \multicolumn{9}{|l|}{ Emotion Subdomain } \\
\hline Happiness & 0.067 & 0.369 & 0.108 & 0.106 & 0.099 & 0.421 & 0.156 & 0.175 \\
\hline Manage stress level & 0.157 & 0.327 & 0.145 & 0.343 & -0.022 & 0.852 & -0.045 & 0.893 \\
\hline Good mood & 0 & 0.65 & 0.048 & 0.584 & 0.121 & 0.286 & 0.078 & 0.477 \\
\hline Positive outlook & 0.18 & 0.053 & 0.145 & 0.106 & -0.066 & 0.647 & -0.056 & 0.525 \\
\hline Calm and peaceful & 0.067 & 0.564 & 0 & 1 & 0 & 0.809 & 0.056 & 0.74 \\
\hline \multicolumn{9}{|l|}{ Self-Image Subdomain } \\
\hline Feel good about self & 0.101 & 0.18 & 0.265 & 0.004 & 0.077 & 0.412 & 0.033 & 0.62 \\
\hline Self-confidence & 0.135 & 0.034 & 0.169 & 0.037 & 0.198 & 0.034 & 0.167 & 0.043 \\
\hline Physical appearance & 0.303 & 0.001 & 0.313 & 0.002 & 0.077 & 0.257 & 0.278 & 0.001 \\
\hline Amount of exercise & 0.124 & 0.552 & 0.145 & 0.19 & 0.135 & 0.145 & 0.133 & 0.175 \\
\hline How clothes fit and look & 0.135 & 0.18 & 0.217 & 0.084 & -0.022 & 0.82 & -0.033 & 0.82 \\
\hline \multicolumn{9}{|l|}{ Self-Competence Subdomain } \\
\hline Ability to take care of self & 0.067 & 0.736 & 0.41 & $<0.001$ & 0.066 & 0.202 & 0.089 & 0.218 \\
\hline Feel confident doing things & 0.303 & 0.009 & 0.386 & 0.003 & 0.143 & 0.172 & 0.122 & 0.213 \\
\hline \multicolumn{9}{|l|}{ Energy and Vitality Domain } \\
\hline \multicolumn{9}{|l|}{ Personal Pleasure Subdomain } \\
\hline Sex life & 0.281 & 0.078 & 0.289 & 0.061 & -0.024 & 0.563 & -0.06 & 0.303 \\
\hline Attend public functions & 0.247 & 0.165 & 0.217 & 0.292 & 0.213 & 0.195 & 0.333 & 0.033 \\
\hline Do enjoyable activities & 0.045 & 0.31 & 0.217 & 0.051 & 0.143 & 0.034 & 0.067 & 0.537 \\
\hline Sports/recreation & 0.034 & 0.291 & -0.265 & 0.138 & 0.164 & 0.171 & -0.081 & 0.635 \\
\hline Hobbies & 0.225 & 0.07 & 0.06 & 0.277 & 0.153 & 0.28 & 0.169 & 0.247 \\
\hline Enjoy friends/family & 0.146 & 0.084 & 0.145 & 0.175 & 0.244 & 0.008 & 0.298 & 0.006 \\
\hline Enjoy time alone & 0.225 & 0.147 & 0.145 & 0.349 & 0.125 & 0.34 & 0.174 & 0.128 \\
\hline \multicolumn{9}{|l|}{ Physical State Subdomain } \\
\hline Feel physically & 0.067 & 0.456 & 0.217 & 0.027 & 0.187 & 0.064 & 0.122 & 0.209 \\
\hline Energy & 0 & 0.605 & 0.157 & 0.111 & 0.286 & 0.01 & 0.189 & 0.184 \\
\hline Felt less tired & 0 & 0.946 & 0.024 & 0.526 & 0.267 & 0.056 & 0.303 & 0.018 \\
\hline Complete household chores & 0.225 & 0.16 & 0.265 & 0.099 & 0.144 & 0.097 & 0.258 & 0.022 \\
\hline \multicolumn{9}{|l|}{ Routine Activity Subdomain } \\
\hline Personal hygiene & 0.011 & 0.97 & 0.072 & 0.503 & 0.143 & 0.036 & 0.067 & 0.401 \\
\hline Go to work or to school & 0.067 & 0.419 & 0.108 & 0.885 & 0.119 & 0.408 & 0.071 & 0.662 \\
\hline Go where want/need to go & -0.101 & 0.359 & 0.036 & 0.922 & 0.146 & 0.049 & 0.011 & 0.805 \\
\hline
\end{tabular}

Note: Participants were asked to fill out the FQoL ${ }^{\mathrm{TM}}$ instrument at three time points: before study commencement (i.e., baseline), after a 4 -week usage period (day 28), and after 2 weeks of product deprivation (day 42). The mean changes from baseline are shown for 28 - and 42 -day time points. The change in the response for each individual item was evaluated for statistical significance using a non-parametric Wilcoxon signed rank test. The 2-tailed $p$ values are reported.

The FQoL ${ }^{\mathrm{TM}}$ instrument was used in a similar study in China among a group of younger cosmetics users (mean age of 25 years, as shown in Table 1). This group showed significant improvement in the mean Overall QoL score and in the Well-Being domain score at both time points, i.e., after 28 days of use and after 5 days of product deprivation (Figure 4a). For the Energy and Vitality domain, the mean scores at 28- and 33-days were virtually unchanged from the baseline value. The increase in the Well-Being domain was driven by directional increases in scores for all three subdomains, i.e., Emotion, Self-Image, and Self Competence (Figure $4 b$ ), however, only the means for the Self-Image subdomain reached significance of $p=0.05$ or less. The subdomains that make up the Energy and Vitality domain were not significantly changed from baseline. 


\section{a. Younger Cosmetics Users - Domains}

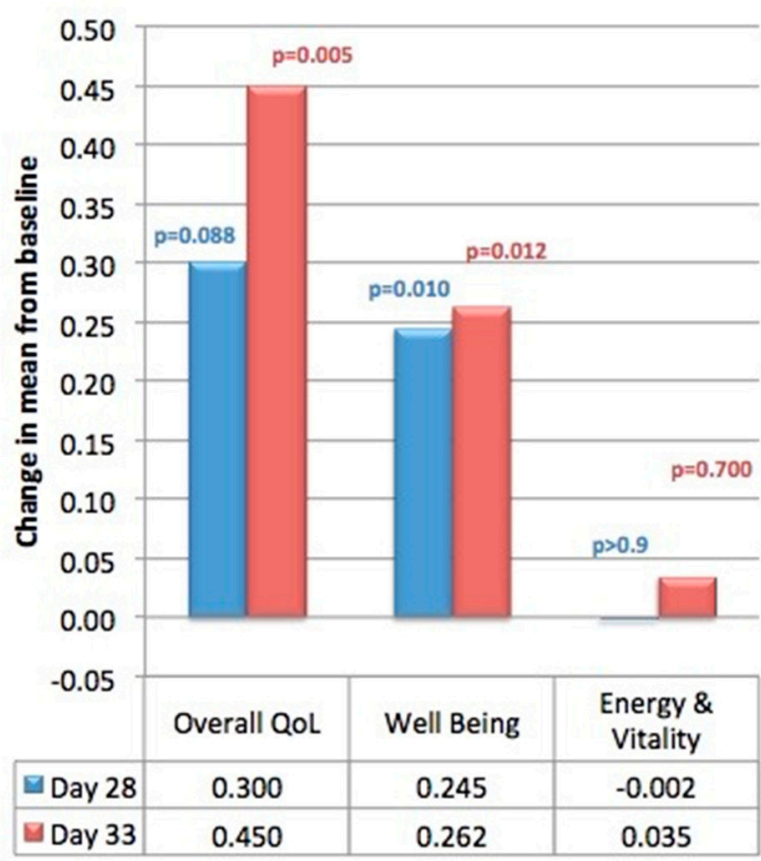

\section{b. Younger Cosmetics Users - Subdomains}

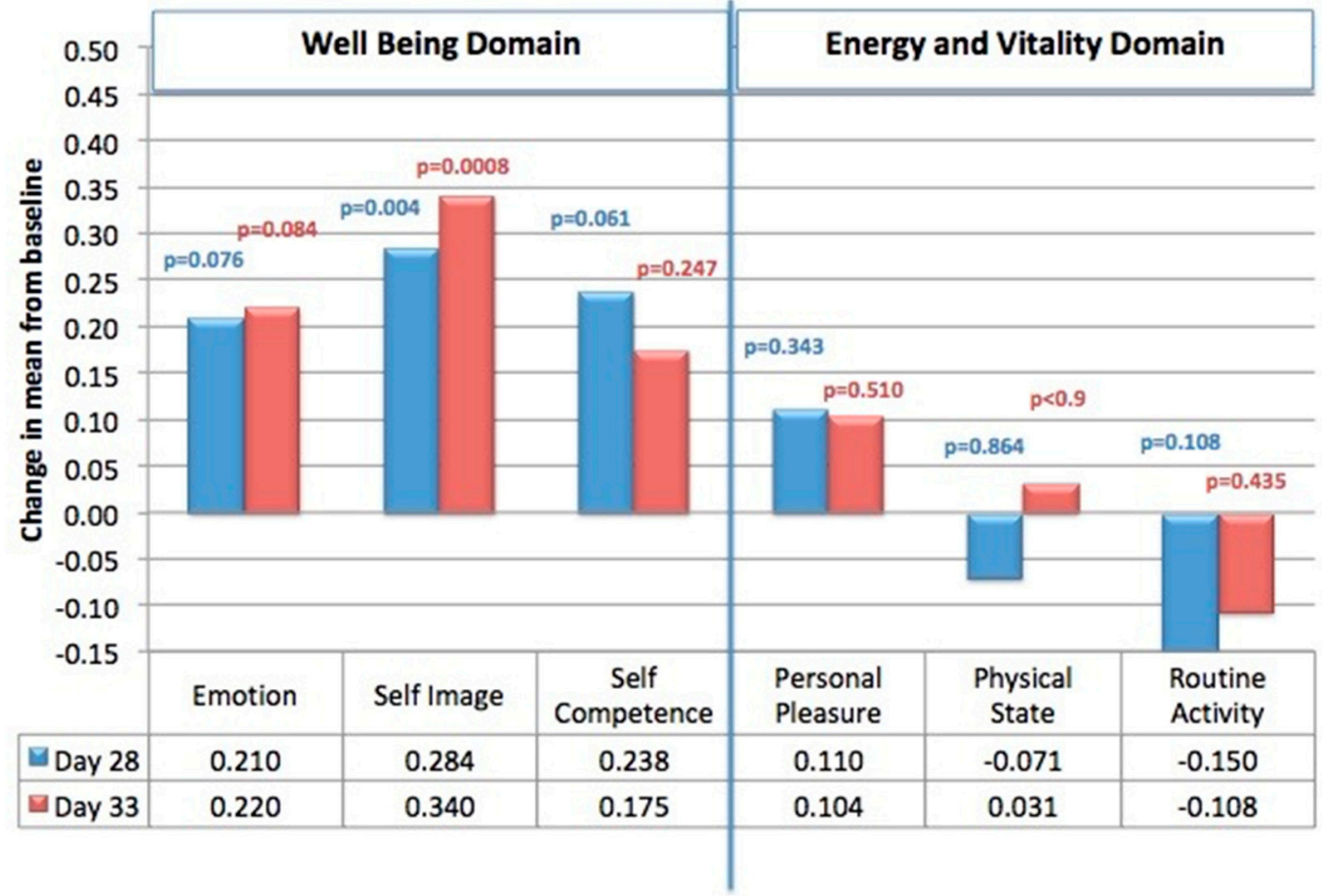

Figure 4. In a study of three parts, skin care regimen participants were asked to fill out the FQoL ${ }^{\mathrm{TM}}$ instrument before and after a 4-week usage period (day 28), and after 5-days of product deprivation (day 33). Mean scores for domains (a) and subdomains (b) were compared to the baseline means for significant changes. Results for the 40 participants in the cohort are presented. 


\section{Discussion}

When comparing the responses of the groups in the Australia study, it must be kept in mind that these two group were significantly different in two respects. First, the mean ages of these groups are decidedly different (Table 1). The average age of the New Mothers group was 34 years old, with more than $90 \%$ of the group less than 40 years (data not shown). The National Representative group had an average age of 45 , with more than $75 \%$ over 34 years of age. Aging skin looks thinner, paler, and dryer, and becomes increasingly more prone to show wrinkles and age spots. Correcting these age-related challenges likely influenced the responses of some individuals in this group. The second substantial difference in the two groups is that mothers with young children find it difficult to find time for self-care. New Mothers often feel that their time is filled with care for their children, and their own appearance is not a priority [22]. In the course of this study the skin care regimen provided the opportunity to spend time on themselves to improve their skin. Additionally, in younger individuals, appearance may be more an important factor in how they present themselves to the outside world, compared to women in the older age group, who may view skin care regimen as something they do for themselves.

Overall, there was little difference between the two groups with regards to questions specific about the condition of their skin in the skin care module (Figure 1). After the product use period both groups of panelists felt the condition of their skin improved feelings of empowerment, happiness, and self-esteem (Figure 1a,b). The New Mothers group (Figure 1a) also responded they felt more confident with less make-up and more attractive, significance in responses was achieved at both 28-day and 42-day time points, respectively. The National Representative group showed significant improvement at both time points regarding feeling attractive, however, they did not improve significantly in feeling confident with less make-up. In addition, one individual item in the standard FQoL ${ }^{\mathrm{TM}}$ that asks specifically about feelings regarding physical appearance, showed significant improvement in both groups (Table 3). This was significant for the New Mothers group at both the 28-day and 42-day time point, and for the National Representative group at the 42-day time point.

The differences between responses of these two groups may be a consequence of the age and responsibilities between the two test groups, and the situation faced by many mothers of young children. Among New Mothers, the improvement in the Self-Image and Self-Competence subdomains was driven by changes directed at themselves, such as feeling good about themselves, and feelings about their physical appearance, feeling confident, and the ability to take care of themselves (Table 3). In general, new mothers tend to spend less time concerned with their own well-being and following the skin care regimen during the study may provide a break from routine childcare and an opportunity to do something that is strictly for themselves. In addition, improvements in the condition of the skin may be much more noticeable among a group that typically has less time for routine skin care. In contrast, the improvement in the Personal Pleasure subdomain among the National Representative group was the result of positive changes in outwardly directed social activities (Table 3). This group felt better about attending functions, engaging in activities, and enjoying time with friends and family.

For many of the individual items in the FQoL ${ }^{\mathrm{TM}}$ instrument (Table 3) and for the skin care module (Figure 1) positive changes lasted or increased between day 28 and day 42, i.e., after the skin care regimen products were withdrawn. This is consistent with the definition of skin care cosmeceuticals as materials having therapeutic effects beyond the duration of application [16]. A separate analysis was conducted comparing the means for the domains and subdomains at day 42 versus day 28 . Increases between day 42 and day 28 were not significant except for the Self Competence domain for the New Mothers (Figure 1b) ( $p=0.021$, data not shown).

The smaller study in China represented a younger age group from a very different cultural environment than the Australia study. The average age in this test group was 25, and the oldest individual was 33 years of age (Table 1). In addition, mothers of young children were excluded from this group. The final FQoL ${ }^{\mathrm{TM}}$ instrument was administered after only 5 days of product withdrawal (on day 33). Unlike both test groups in the Australia study, this group showed a significant improvement in the mean score for overall QoL at day 33 (Figure 4a). Neither group in the Australia study showed 
significant improvement in this item at either time point. The test group in China also showed significant improvement in the Well-Being domain (Figure 4a), with significant positive changes in the Self-Image subdomain (Figure $4 b, p=0.004$ and 0.0008 at 28- and 33-days, respectively). The skin care module was not included in this study; however, this group exhibited a significant improvement in the physical appearance item after the 28-day product usage phase (mean improvement in score $=0.325$, $p$ value $=0.031$, data not shown) .

One limitation of the use of QoL to evaluate the effects of consumer products is the mindset shift needed in terms of the changes, which will likely be incrementally small. QoL instruments have been used predominantly to evaluate the impact of chronic disease states, the effectiveness of specific medications, or the potential positive results from different therapeutic approaches. In these cases, the patients' quality of life generally starts lower than the average individual not suffering from similar maladies, and the QoL attempts to evaluate improvement toward that of the average individual. We have used the FQoL instrument to evaluate the effects of consumer products on generally healthy individuals who are not suffering from any specific disease states. In other words, our subjects start at "average QoL" and we are attempting to measure further increases. As a result, changes in QoL could be expected to be relatively small. Despite that, we are able to see differences that reached significance.

Investigations have shown that physical attractiveness is an important determinant of interpersonal relationships, especially in the early stages [23]. The use of make-up is widely portrayed as a tool for improving facial attractiveness. Korichi and colleagues developed a questionnaire to evaluate the reasons women used make-up [23]. Investigations have shown that women use cosmetics for a variety of reasons ranging from feeling less self-consciousness about their appearance, feeling more assertive and confident, or appearing healthier [23,24]. A skin care regimen can have a positive impact on the QoL of consumers. This study demonstrates that routine skin care with quality products that are effective in addressing skin concerns also contributes to improving self-esteem, and has positive effects on several aspects of a person's self-image. Differences in responses of the test groups were likely related to differences in the mean age and differences in time available to look after themselves.

Author Contributions: Set-up \& executed the study with a third party agency in Australia: L.Z., A.A. and J.Q. in China. Design consultant for both studies: M.W. All statistical analysis and critical review of the manuscript: P.S. Methodology, conceptualization and visualizations: M.A.F. Lead writing, editing, and critical review of manuscript: M.A.F., A.A., L.Z. Project sponsors: V.S., R.L., M.S. All authors have read and agreed to the published version of the manuscript.

Funding: This work was funded by The Procter and Gamble Company.

Acknowledgments: The authors thank Terresa L. Nusair; (Health and Environmental Safety Alliance, Cincinnati, $\mathrm{OH}, \mathrm{USA})$ for technical input.

Conflicts of Interest: Declare conflicts of interest or state "All authors are employees of the Procter and Gamble Company".

\section{References}

1. PROQOLIDtm. eProvide: Online Support for Clinical Outcome Assessments. Available online: https: //eprovide.mapi-trust.org/about/about-proqolid (accessed on 18 February 2020).

2. Rajiah, K.; Maharajan, M.K.; Yeen, S.J.; Lew, S. Quality of Life and Caregivers' Burden of Parkinson's Disease. Neuroepidemiology 2017, 48, 131-137. [CrossRef] [PubMed]

3. El-Jawahri, A.; Greer, J.A.; Pirl, W.F.; Park, E.R.; Jackson, V.A.; Back, A.L.; Kamdar, M.; Jacobsen, J.; Chittenden, E.H.; Rinaldi, S.P.; et al. Effects of Early Integrated Palliative Care on Caregivers of Patients with Lung and Gastrointestinal Cancer: A Randomized Clinical Trial. Oncologist 2017, 22, 1528-1534. [CrossRef] [PubMed]

4. Ribe, J.M.; Salamero, M.; Perez-Testor, C.; Mercadal, J.; Aguilera, C.; Cleris, M. Quality of life in family caregivers of schizophrenia patients in Spain: Caregiver characteristics, caregiving burden, family functioning, and social and professional support. Int. J. Psychiatry Clin. Pract. 2018, 22, 25-33. [CrossRef] [PubMed]

5. Chernyshov, P.V. The Evolution of Quality of Life Assessment and Use in Dermatology. Dermatology 2019, 235, 167-174. [CrossRef] [PubMed] 
6. Seidler, A.M.; Bayoumi, A.M.; Goldstein, M.K.; Cruz, P.D.J.; Chen, S.C. Willingness to pay in dermatology: Assessment of the burden of skin diseases. J. Investig. Dermatol. 2012, 132, 1785-1790. [CrossRef] [PubMed]

7. Beikert, F.C.; Langenbruch, A.K.; Radtke, M.A.; Augustin, M. Willingness to pay and quality of life in patients with rosacea. J. Eur. Acad. Dermatol. Venereol. 2013, 27, 734-738. [CrossRef] [PubMed]

8. Beikert, F.C.; Langenbruch, A.K.; Radtke, M.A.; Kornek, T.; Purwins, S.; Augustin, M. Willingness to pay and quality of life in patients with atopic dermatitis. Arch. Dermatol. Res. 2014, 306, 279-286. [CrossRef] [PubMed]

9. Beresniak, A.; de Linares, Y.; Krueger, G.G.; Talarico, S.; Tsutani, K.; Duru, G.; Berger, G. Validation of a new international quality-of-life instrument specific to cosmetics and physical appearance: BeautyQoL questionnaire. Arch. Dermatol. 2012, 148, 1275-1282. [CrossRef] [PubMed]

10. Chang, A.L.S.; Chen, S.C.; Osterberg, L.; Brandt, S.; von Grote, E.C.; Meckfessel, M.H. A daily skincare regimen with a unique ceramide and filaggrin formulation rapidly improves chronic xerosis, pruritus, and quality of life in older adults. Geriatr. Nurs. 2018, 39, 24-28. [CrossRef] [PubMed]

11. Zhuang, X.S.; Zheng, Y.Y.; Xu, J.J.; Fan, W.X. Quality of life in women with female pattern hair loss and the impact of topical minoxidil treatment on quality of life in these patients. Exp. Ther. Med. 2013, 6, 542-546. [CrossRef] [PubMed]

12. Getliffe, K.; Fader, M.; Cottenden, A.; Jamieson, K.; Green, N. Absorbent products for incontinence: 'treatment effects' and impact on quality of life. J. Clin. Nurs. 2007, 16, 1936-1945. [CrossRef] [PubMed]

13. Farage, M.A.; Nusair, T.L.; Hanseman, D.; Sherman, S.N.; Tsevat, J. The Farage Quality of Life Measure for Consumer Products: Development and Initial Implementation. Appl. Res. Qual. Life 2010, 5, 1-25. [CrossRef]

14. Tzeghai, G.E.; Ajayi, F.O.; Miller, K.W.; Imbescheid, F.; Sobel, J.D.; Farage, M.A. A Feminine Care Clinical Research Program Transforms Women's Lives. Glob. J. Health Sci. 2015, 7, 45-59. [CrossRef] [PubMed]

15. Garcia, A. The Skincare Industry Is Booming, Fueled by Informed Consumers and Social Media. Available online: https://www.cnn.com/2019/05/10/business/skincare-industry-trends-beauty-social-media/index.html (accessed on 24 January 2020).

16. Pandey, A.; Jatana, G.K.; Sonthalia, S. “Cosmeceuticals.” StatPearls. Available online: https://www.ncbi.nlm. nih.gov/pubmed/31334943 (accessed on 10 February 2020).

17. Matts, P.J.; Oblong, J.E.; Bissett, D.L. A review of the range of effects of niacinamide in human skin. IFSCC Mag. 2002, 5, 285-289.

18. Bissett, D.L.; Miyamoto, K.; Sun, P.; Li, J.; Berge, C.A. Topical niacinamide reduces yellowing, wrinkling, red blotchiness, and hyperpigmented spots in aging facial skin. Int. J. Cosmet. Sci. 2004, 26, 231-238. [CrossRef]

19. WHO. World Health Organization Quality of Life (WHOQOL). Available online: https://www.who.int/ mental_health/publications/whoqol/en/ (accessed on 13 July 2020).

20. Pratt, J.W. Remarks on Zeros and Ties in the Wilcoxon Signed Rank Procedures. J. Am. Stat. Assoc. 1959, 54, 655-667. [CrossRef]

21. Farage, M.A.; Rodenberg, C.; Chen, J. Translation and Validation of the Farage Quality of Life (FQoL) Instrument for Consumer Products into Traditional Chinese. Glob. J. Health Sci. 2013, 5, 1-12. [CrossRef]

22. Cameron, S. Unexplainable Feelings Every New Mom Has. Available online: https://www.babygaga.com/15unexplainable-feelings-every-new-mom-has/ (accessed on 27 January 2020).

23. Korichi, R.; Pelle-de-Queral, D.; Gazano, G.; Aubert, A. Why women use makeup: Implication of psychological traits in makeup functions. J. Cosmet. Sci. 2008, 59, 127-137. [PubMed]

24. Jones, A.L.; Kramer, R.S. Facial Cosmetics and Attractiveness: Comparing the Effect Sizes of ProfessionallyApplied Cosmetics and Identity. PLoS ONE 2016, 11, e0164218. [CrossRef] [PubMed]

(C) 2020 by the authors. Licensee MDPI, Basel, Switzerland. This article is an open access article distributed under the terms and conditions of the Creative Commons Attribution (CC BY) license (http://creativecommons.org/licenses/by/4.0/). 